\title{
Effect of density functionals on the vibrational and thermodynamic properties of $\mathrm{Fe}_{2} \mathrm{VAl}$ and $\mathrm{Fe}_{2}$ TiSn compounds
}

\author{
Shivprasad S. Shastr: ${ }^{*}$ and Sudhir K. Pandey \\ School of Engineering, Indian Institute of Technology Mandi, Kamand - 175005, India
}

(Dated: June 18, 2018)

\begin{abstract}
First-principles phonon calculations along with Kohn-Sham density functional theory (DFT) is an essential tool to study the lattice dynamics, thermodynamical properties and phase-transitions of materials. The two full-Heusler compounds $\mathrm{Fe}_{2} \mathrm{VAl}$ and $\mathrm{Fe}_{2} \mathrm{TiSn}$ are studied for lattice vibration dependent properties using finite displacement method and supercell approach. For the investigation, four density functionals viz., LDA, PBE, PBEsol and meta-GGA SCAN are employed. Using these functionals, phonon dispersion, phonon density of states (DOS), partial density of states (PDOS) thermal propertis and zero-point energy are calculated at equilibrum lattice parameters under harmonic approximation. For the two compounds the Debye temperatures are calculated from the obtained phonon DOS which are $\sim 660 \mathrm{~K}$ and $\sim 540 \mathrm{~K}$, respectively. The obtained results from different functionals are compared among each other. The overall phonon energy in the dispersion is found to be $\sim 15 \mathrm{meV}$ higher in $\mathrm{Fe}_{2} \mathrm{VAl}$ than the $\mathrm{Fe}_{2} \mathrm{TiSn}$ compounds. For the two compounds $\mathrm{PBE}$ is yielding the lowest phonon frequencies while LDA or SCAN functional is giving the highest. The same pattern is observed in phonon DOS plots of two compounds. The zero-point energy calculated is the highest from SCAN (21.04 and 16.95 J) and the lowest from PBE functionals (20.09 and $16.02 \mathrm{~J}$ ) obeying the same trend as frequency for both compounds. A general prediction of nature of lattice thermal conductivity is made based on the velocities of acoustic phonons which is in agreement with the qualitative behavior of reported experimental thermal conductivity of two compounds. Phonon spectra obtained from PBE and SCAN have similar general features while those from LDA and PBEsol have resembling features for $\mathrm{Fe}_{2} \mathrm{VAl}$, while this trend is not observed for the compound $\mathrm{Fe}_{2} \mathrm{TiSn}$.

Key words: Phonons, exchange-correlation functionals, lattice thermal conductivity, lattice dynamics.
\end{abstract}

\section{INTRODUCTION}

First-principles Kohn-Sham density functional theory $(\mathrm{DFT})^{\frac{1}{}}$ is one of the most widely used theoretical investigation tools in materials science. This tool has been very helpful since it's inception for the study of electronic structure, and other ground-state properties. This is also made possible with the aid of advancements in the field of high-perfomance computing and it's cost effectiveness. ${ }^{2}$ In the KS-DFT the total ground-state enrergy is calculated as a functional of the ground-state electron density for a static lattice and is minimized with respect to lattice constant. The total ground state energy is the sum of kinetic energy, Coulomb energy and exchange-correlation (XC) energy. And this XC energy is the part which is approximated in practice $\frac{3}{-}$ So, the best explanation to experimental results from DFT calculations depends on the approximation used in $\mathrm{XC}$ functionals. There are large number of approximations available to XC energy. LDA and GGA-PBE are the mostly used density functionals.

This application of first-principles calculations is extended in the field of condensed matter physics and materials science by the introduction of phonon calclations $2.4,5$ When an atom is displaced from the equilibrium position in a crystal forces arise on all the atoms. By calculating the force on the each atom using the total energy of the crystal, phonon frequencies can be obtained. This approach of calculation is called finitedisplacement method (FDM). Another method uses den- sity functional perturbation theory (DFPT) to calculate the phonon frequencies and hence dependent properties $\underline{\underline{4}}$

$\mathrm{Fe}_{2} \mathrm{VAl}$ and $\mathrm{Fe}_{2}$ TiSn are the two compounds belonging to the family of full-Heusler alloys. These compounds are reported to have non-magnetic ground state with applications in many fields. They crystallise in cubic $L 22_{1}$ phase with space group $F m-3 m \underline{\underline{6}} \underline{\underline{-8}}$ The two compounds are also being studied for the application as thermoeletrics for heat energy conversion $\stackrel{9,10}{ } \mathrm{~A}$ thermoelectric is characterized by it's figure of metit (ZT) value. As it is well known that ZT is proportional to the electrical conductivity and inversly related to thermal conductivity, it is important to study the electronic structure of a material in order to understand or improve the efficiency $\stackrel{11,12}{10}$ Since, the thermal conductivity has an important contribution from lattice vibrations, it is must to investigate the lattice thermal conductivity if one has to properly understand a thermoelectric in order to tailor it's efficiency $\stackrel{12}{\underline{12}}$

Keeping this in mind, we have studied the electronic strucure of the two compounds employing five XC functionals in our recently reported study $\underline{\underline{13}}$ We also calculated effective masses for two compounds and found that $\mathrm{Fe}_{2}$ TiSn showing large value of effective mass having flat band. In this direction, to completely understand the thermoelectric behavior, there is a need to study the lattice part of thermal conductivity of the two compounds through phonon calculations. Also, the lattice dynamics study of the two compounds in literature using different functionals is missing. Performing the test of functionals 
to study phonon frequencies along with electronic structure gives strict test of the functional. 2

Therefore, in the present work, we have undertaken the study of two full-Heusler compounds, $\mathrm{Fe}_{2} \mathrm{VAl}$ and $\mathrm{Fe}_{2}$ TiSn using four XC functionals. The XC functionals employed are, LDA-PW92 ${ }^{14}$, GGA-PBE ${ }^{15}$, PBEsol ${ }^{16}$ and SCAN ${ }^{17}$ meta-GGA. Using these functionals phonon dispersion, density of states (DOS), partial density of sates (PDOS) are calculated at equilibrium lattice parameters under harmonic approximation. Further, variation in thermodynamical quantities, constant volume specific heat $C_{V}$ and Helmholtz free energy (F) as a function of temperature are calculated. The Debye temeperature and the zero-point energy contribution to the static lattice are also calculated. The effect of the various functionals on the obtained phonon properties are discussed. Lattice thermal conductivity prediction from the slope of acoustic phonon branches are guessed. And this result is compared qualitatively with the experimental thermal conductivity study of two compounds.

\section{COMPUTATIONAL DETAILS}

In this work calculations are carried out using two computational programs. To obtain the total forces on the atoms , first-principles full-potential linearized augmented plane wave (FP-LAPW) based program WIEN2 $\mathrm{k}^{18}$ is used as force calculator. Self-consistent cycles are run till the sum of total forces on atoms is less than $0.1 \mathrm{mRy} / \mathrm{bohr}$. The calculations are performed using four exchange-correlation functionals viz., $\mathrm{LDA}^{14}, \mathrm{PBE}^{15}, \mathrm{PBEso}^{16}$ and recent meta-GGA functional SCAN $\stackrel{17}{17} \mathrm{k}$-mesh of size $5 \times 5 \times 5$ is used for both $\mathrm{Fe}_{2} \mathrm{VAl}$ and $\mathrm{Fe}_{2} \mathrm{TiSn}$ compounds for force calculation. For the phonon calculations part PHONOPY ${ }^{4}$ code is used. PHONOPY $\underline{\underline{4}}$ is a first-principles phonon calculations tool which can handle force constants obtained from both finite displacement method (FDM) and density functional pertrubation theory (DFPT) for phonon frequency analysis. Here, FDM and supercell approach is used to calculate phonon properties. A supercell of size $2 \times 2 \times 2$ of primitive lattice type is constructed for both the compounds with 128 atoms. The supercell is used to obtain the long-range force constants between atoms $\stackrel{19}{19} \mathrm{An}$ artificial force is created in the systems by displacing atom of each kind ( $\mathrm{Fe}, \mathrm{V}, \mathrm{Al}, \mathrm{Ti}, \mathrm{Sn})$ in the formula units by a distance of $0.02 \mathrm{bohr}$ in x-direction. Further analysis of total forces on atoms to get forcecosntants and post-processing of them are carried out using PHONOPY ${ }^{4}$ to obtain phonon dispersions and phonon density of states (DOS) and thermal properties on a sampling mesh of $21 \times 21 \times 21 . \mathrm{Fe}_{2} \mathrm{VAl}$ and $\mathrm{Fe}_{2} \mathrm{TiSn}$ full-Heusler compounds have cubic $L 2_{1}$ crystal structure with Fm-3m space group. The Fe atoms occupy the Wyckoff position 8c $\left(\frac{1}{4}, \frac{1}{4}, \frac{1}{4}\right), \mathrm{Y}$ atoms occupy Wyckoff position $4 \mathrm{a}(0,0,0)$ and $\mathrm{Z}$ atoms occupy Wyckoff position $4 \mathrm{~b}\left(\frac{1}{2}, \frac{1}{2}, \frac{1}{2}\right) \stackrel{\underline{20}}{\underline{\underline{2}}}$ Where $\mathrm{Y}=\mathrm{V}, \mathrm{Ti}$ and $\mathrm{Z}=\mathrm{Al}, \mathrm{Sn}$ in the general representation $\mathrm{Fe}_{2} \mathrm{YZ}$ of these two full-Heusler compounds $\underline{13}$

\section{RESULTS AND DISCUSSION}

The lattice vibration dependent properties of the two Heusler compounds are studied under harmonic approximation at equilibrium lattice parameters. The equilibrium lattice constants are obtained by minimizing the total energy with volume. The obtained optimized lattice constant values from four different XC functionals are presented in Table 1 . The details of the volumeoptimization calculation can be found in our previous work $\underline{\underline{13}}$

From the Table $1: \underline{\underline{13}}$ the trends in the obtained equilibrum lattice parameters from four functionals can be observed. In these cases also the overbinding nature of LDA and relatively underbinding nature of PBE are clearly exhibited with the lowest and highest values of lattice constants, respectively.

To further study the lattice stability, phonon energy and thermodynamic properties of the two full Heusler compounds, first-principles phonon calculations are carried out.

\section{A. Vibrational properties}

The phonon band structure and density of states (DOS) and partial density of states (PDOS) are calculated for the two compounds using four XC functionals. The corresponding phonon band structures from the four functionals for $\mathrm{Fe}_{2} \mathrm{VAl}$ and $\mathrm{Fe}_{2} \mathrm{TiSn}$ are shown in Fig. 1(a)-(d) and (e)-(h), respectively. The vibrational spectra is plotted along $\Gamma-X-W-\Gamma-L$ directions in the first Brillouin zone. A crystal is considered to be mechanically stable if the potential energy of the crystal increases for any displacements of atoms inside it. According to harmonic approximation, this condition is satisfied when all the phonons have real and positive frequencies (or energies) $\underline{\underline{4}}$ The negative or imaginary frequencies imply that the crystal is dynamically unstable discarding the possibility of synthesis in that phase. In our case, the obtained dispersion curves for two compounds does not show any negative frequecies indicating that both the compounds are stable in the cubic $L 2_{1}$ phase. The two branches out of the total three acoustic branches are doubly degenerate (or nearly doubly degenerate) nearly halfway along $\Gamma-W$ direction and fully along $\Gamma-L$ direction in case of both the compounds. The acoustic branches of $\mathrm{Fe}_{2} \mathrm{VAl}$ are following the linear relation with $\mathbf{q}$ for more distance (i.e more shorter wavelength) than in the case $\mathrm{Fe}_{2}$ TiSn, along both $\Gamma-W$ and $\Gamma-L$ directions. In this linear region the group velocity will be the same as phase velocity $\underline{21}$ Few of the acoustic and optical branches of $\mathrm{Fe}_{2} \mathrm{VAl}$ are degenerate at an energy of $\sim 29 \mathrm{meV}$ and $\sim 33$ $\mathrm{meV}$, respectively at the zone boundary containg point 
TABLE I: Calculated lattice constants $a_{0}$ for the two compounds using five exchange-correlational functionals

\begin{tabular}{ccc}
\hline \hline & $\mathbf{F e}_{2} \mathbf{V A l}$ & $\frac{\mathbf{F e}_{2} \mathbf{T i S n}}{}$ \\
\cline { 2 - 3 } & $\begin{array}{c}\text { Lattice constant }\left(a_{0}\right) \\
\end{array}$ & $\begin{array}{c}\text { Lattice constant }\left(a_{0}\right) \\
(\AA)\end{array}$ \\
\hline LDA & 5.5955 & 5.9102 \\
PBE & 5.7089 & 6.0423 \\
PBEsol & 5.6478 & 5.9664 \\
SCAN & 5.6509 & 5.9762 \\
\hline \hline
\end{tabular}

$W$. But this nature is not observed in case of $\mathrm{Fe}_{2} \mathrm{TiSn}$ vibrational spectrum, where there is a clear seperation between optical and acoustic phonon branches. On observing the energy scales of the dispersion curves of the two compounds, it is visible that the vibrational energy of phonons is more in $\mathrm{Fe}_{2} \mathrm{VAl}$ than in the case of $\mathrm{Fe}_{2} \mathrm{TiSn}$ compound. The $\mathrm{Fe}_{2}$ TiSn compound contains heavier element $\mathrm{Sn}$ and it's atomic mass is more than the $\mathrm{Fe}_{2} \mathrm{VAl}$ compound. As the vibrational frequency, in turn energy, is inversly proportional to the suare root of mass of elements in the compound, these observations justify the frequency-mass relation. In the $\mathrm{Fe}_{2} \mathrm{VAl}$ vibrational spectrum we observe three acoustic branches are crossing with some of the optical branches. And there are three high energy optical branches of $\sim 50 \mathrm{meV}$ with a distinct seperation from the other branches. In $\mathrm{Fe}_{2} \mathrm{VAl}$, looking at the atomic masses of the constituents of the formula unit, $\mathrm{Al}$ atom has the lowest atomic mass. Therefore, the major contribution to these curves should be from that atom ${ }^{22}$ The crossing branches in the region between $\sim 25-40$ meV energy are mainly due to the near atomic mass elements $\mathrm{Fe}$ and $\mathrm{V}$. Now, while going from $\mathrm{Fe}_{2} \mathrm{VAl}$ to $\mathrm{Fe}_{2} \mathrm{TiSn}$ the following changes in the vibrational spectrum of the latter compound are observed. The highest energy optical phonon branches are observed $\sim 35 \mathrm{meV}$ in the energy scale and which is shifted down compared to that of $\mathrm{Fe}_{2} \mathrm{VAl}$. Compared with the former compound here, three lower energy acoustic branches are found to be well seperated from the higher energy optical phonon disperion curves except at the $L$-point with energy difference of $\sim 1 \mathrm{meV}$. In this case, the major contribution to the energy of these acoustic phonons are from the heavier atomic mass element $\mathrm{Sn}$ in the compound. The contribution to the optical branches in sim25-40 meV regions are maninly due to the near atomic mass elements $\mathrm{Fe}$ and $\mathrm{Ti}$ atoms in $\mathrm{Fe}_{2} \mathrm{TiSn}$. These qualitative explanations are justified further through phonon PDOS plots in figure 3. As mentioned before, the linear relationship bewteen frequency (or energy) and $\mathbf{q}$ of acoustic branches is followed for small values of $\mathbf{q}$ in $\mathrm{Fe}_{2} \mathrm{TiSn}$ compared to that in the dispersion relation of $\mathrm{Fe}_{2} \mathrm{VAl}$. With the seperation between acoustic and optical phonons mentioned, the degeneracy observed among them in case of the former compound is absent here. One of the in- teresting features of the vibrational spectra of the two compounds can be highlighted here. That is, on examining the slopes of the acoustic branches of the two compounds, the slope values in the linear region are decreasing from $\mathrm{Fe}_{2} \mathrm{VAl}$ to $\mathrm{Fe}_{2} \mathrm{TiSn}$. It is known that, slopes of the acoustic branches are associated with sound velocity and in turn, give the group velocity (and phase velocity in linear region). Also, lattice thermal conductivity in a solid is directly proportional to the sound velocity 21,23 So, knowing the value of sound velocity one can proceed to calculate lattice thermal conductivity. Qualitatively, thus it can be said that lattice thermal conductivity is higher in $\mathrm{Fe}_{2} \mathrm{VAl}$ compared to $\mathrm{Fe}_{2} \mathrm{TiSn}$ which is in the context of thermoelectric application is a useful information. Lue et. al have reported the thermoelectric properties of Heusler compounds $\mathrm{Fe}_{2-x} \mathrm{~V}_{1+x} \mathrm{M}(\mathrm{M}=\mathrm{Al}, \mathrm{Ga}) \stackrel{10}{\underline{10}}$ and $\mathrm{Fe}_{2-x} \mathrm{Ti}_{1+x} \mathrm{Sn} . \underline{\underline{9}}$ The reported value of thermal conductivity is higher in case of $\mathrm{Fe}_{2} \mathrm{VAl}$ than in the $\mathrm{Fe}_{2} \mathrm{TiSn}$ compound under the temperature range studied. Also, in both the compounds they have suggested total thermal conductivity is essentially from lattice part of thermal conductivity. These experimental results are in support of the qualitative explanation given above from our study of the two compounds with sound velocity higher in the former compound than in the $\mathrm{Fe}_{2} \mathrm{TiSn}$ as stated before. Thus, generally, based on the phonon disprersion analysis of this class of compounds we can say that going for a higher atomic mass compounds in the series can leads to lower value of lattice thermal conductivity keeping in mind the corresponding electronic structure of the compound with the supporting data of experimental thermal conductivity from $\mathrm{Fe}_{2} \mathrm{VAl}, \mathrm{Fe}_{2} \mathrm{VGa}^{10}, \mathrm{Fe}_{2} \mathrm{TiSn}$

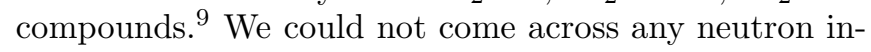
elastic scattering experimental data of phonon dispersion of both the compounds to compare the vibrational sperctrum of our study. Thus, it would be interesting to study the two compounds experimentally to compare the phonon dispersion results obtained from the calculations.

The phonon density of states (DOS) plots for two compounds are presented in Fig. 2 (a) and (b) from all four functionals under study. In Fig. 2(a), phonon DOS of $\mathrm{Fe}_{2} \mathrm{VAl}$ can be seen. Corresponding to the crossing branches of acoustic and optical phonons of the compound high value of phonon DOS peak can be seen in 

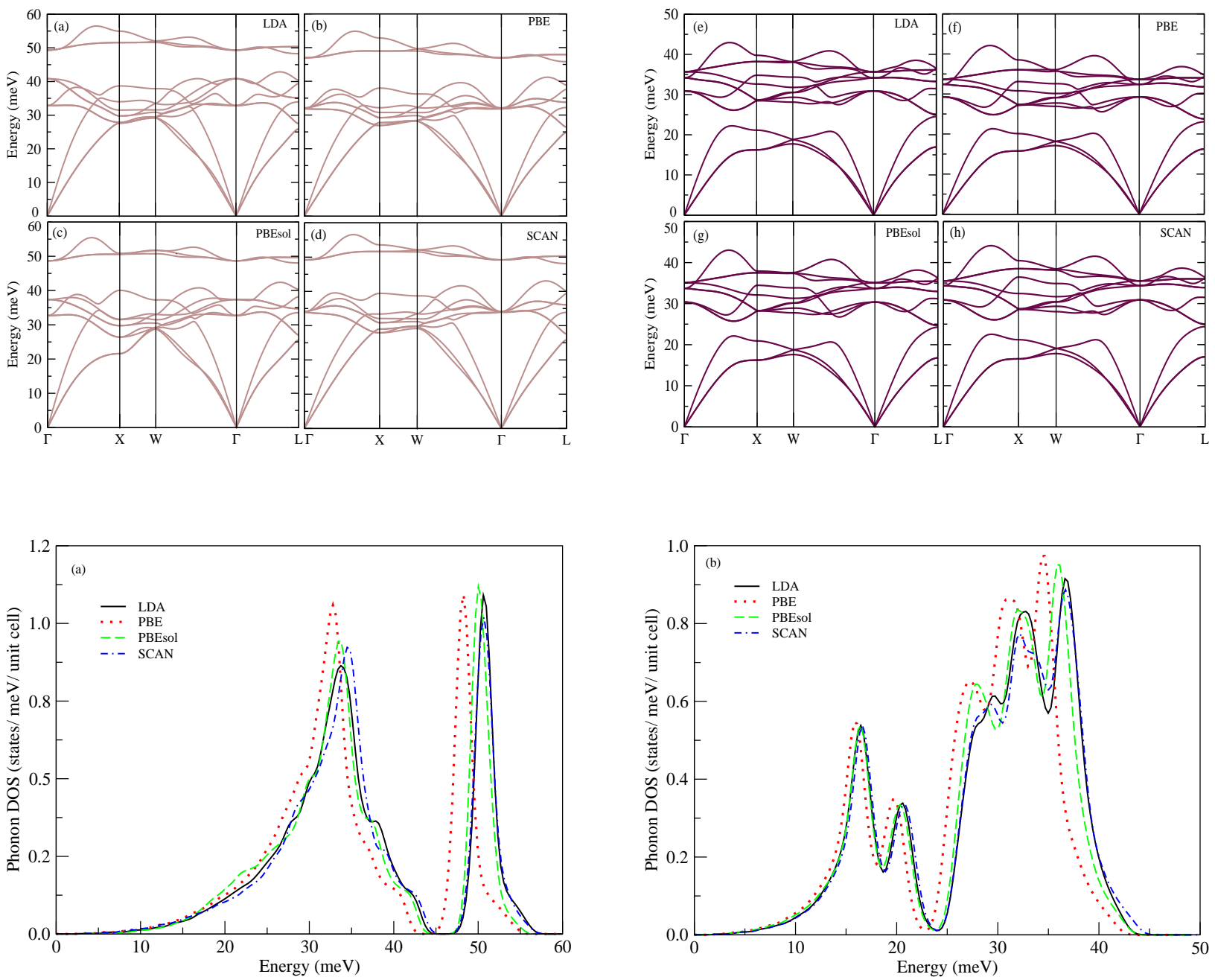

FIG. 2: Phonon DOS for (a) $\mathrm{Fe}_{2} \mathrm{VAl}$ and (b) $\mathrm{Fe}_{2} \mathrm{TiSn}$ compounds

$\sim 25-40 \mathrm{meV}$ region. The highest energy DOS peak in the neighborhood of $50 \mathrm{meV}$ is representing the seperated optical branches in the spectra which is contributed mainly from $\mathrm{Al}$ vibrational states ${ }^{22}$ Vibrational DOS of $\mathrm{Fe}_{2}$ TiSn compound is shown in Fig. 2(b). Clearly, the two peaks in the lower energy region correspond to the three acoustic branches in the disperion plot. The next seperated higher energy peaks represent the coupled optical phonon branches. The nature of the respective phonon DOS peaks justifies the phonon dispersion of the two compounds shown in Fig. 1.

To see the contribution to the phonon frequencies from the different atoms, phonon partial density of states are calculated. Fig. 3 (a)-(d) shows the partial DOS for $\mathrm{Fe}_{2} \mathrm{VAl}$ obtained from four functionals. It is known that, $\mathrm{Al}$ is the lighter element compared to $\mathrm{Fe}$ and $\mathrm{V}$. In the $\sim 20-40 \mathrm{meV}$ region the main contribution to DOS is from $\mathrm{Fe}$ and $\mathrm{V}$ atoms. It can be observed that the higher energy three optical branches has primary contribution from $\mathrm{Al}$ atom. For $\mathrm{Fe}_{2}$ TiSn compound, the phonon partial DOS plot is given in Fig. $3(\mathrm{e})-(\mathrm{h})$. In the plot the acoustic phonons belong to the energy upto $\sim 25 \mathrm{meV}$.
As we can see, energies of acoustic phonon branches are cheifly from the heavier Sn atoms. Contribution to DOS from $\mathrm{Fe}$ and $\mathrm{Ti}$ atoms are less in this region. The PDOS plot also shows for $\mathrm{Fe}_{2} \mathrm{TiSn}$, the optical phonons are contributed from the lighter $\mathrm{Fe}$ and $\mathrm{Ti}$ atoms. In both the compounds, the lighter atoms $\mathrm{Al}$ and $\mathrm{Ti}$ are contributing less in the lower frequency region and more in higher frequency region to the DOS, respectively.

\section{B. Thermal properties}

Using the thermodynamic equations, Helmholtz free energy $(F)$ and constant volume specific heat $\left(C_{V}\right)$ at different temperatures are calculated from PHONOPY $\underline{4}$ The results obtained from all the four functionals for the two compounds are shown in Fig.4 (a) and (b), respectively. In the figure the dotted line repersents the classical Dulong and Petit value of $C_{V}$, which is in this case $\sim 100 \mathrm{~J} / \mathrm{K} / \mathrm{mol}$. As can be seen from the Fig. 4(a) and (b), in case of $\mathrm{Fe}_{2} \mathrm{VAl}$, the Dulong and Petit limit of $C_{V}$ is 

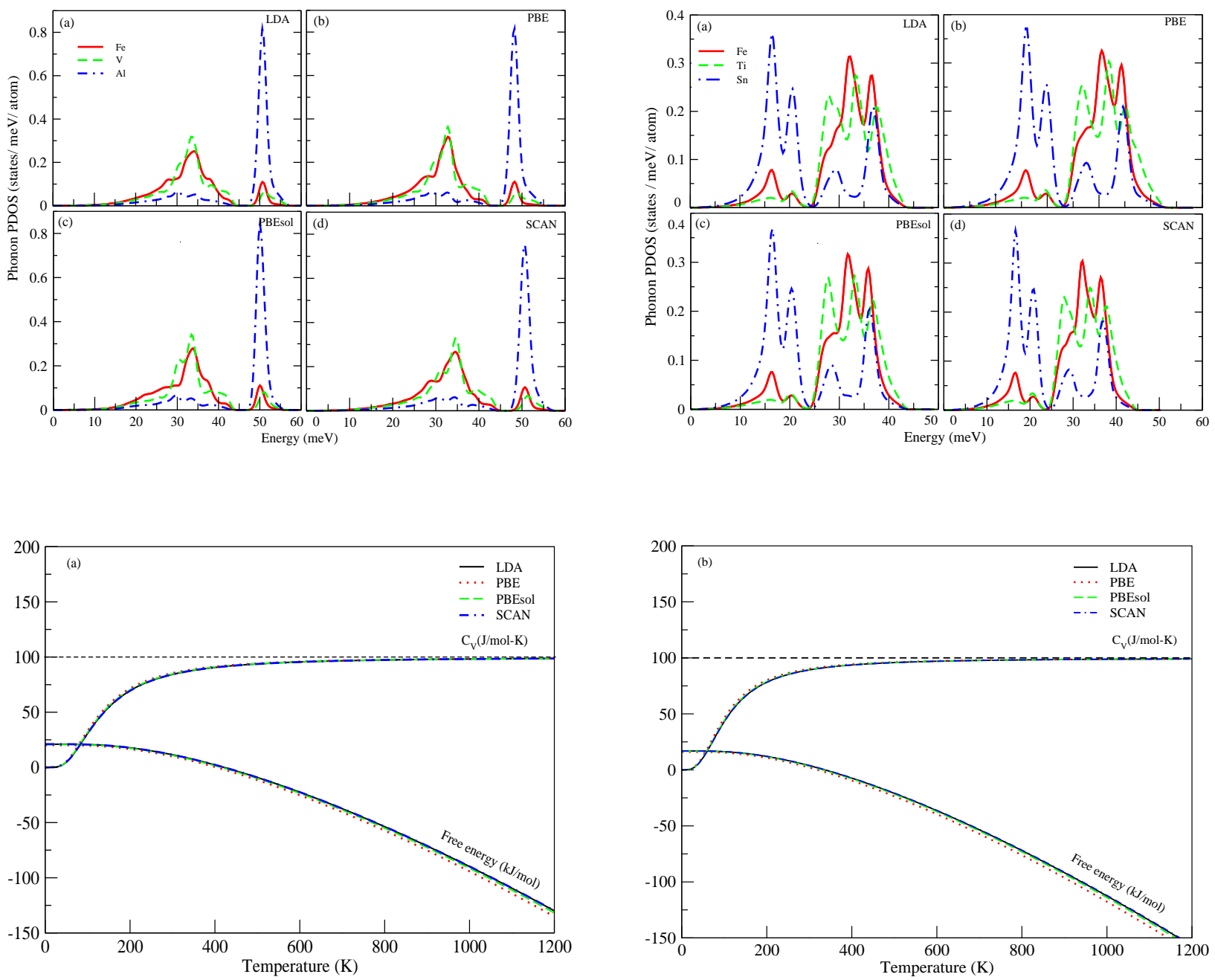

FIG. 4: Free energy (F) and constant volume specific heat $\left(C_{V}\right)$ for (a) $\mathrm{Fe}_{2} \mathrm{VAl}$ and (b) $\mathrm{Fe}_{2} \mathrm{TiSn}$ compounds

reaching at $\sim 650 \mathrm{~K}$. But, for $\mathrm{Fe}_{2} \mathrm{TiSn}, C_{V}$ is approaching the Dulong and Petit value at $~ 550 \mathrm{~K}$. Employing

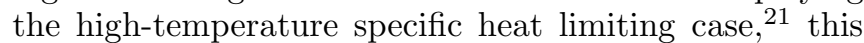
can be justified with the obtained higher energy (or frequency) of phonons in case of $\mathrm{Fe}_{2} \mathrm{VAl}$ and lower energy of phonons in case of $\mathrm{Fe}_{2} \mathrm{TiSn}$ band structures, respectively. The variation of $\mathrm{F}$ at different temperatures are also shown in Fig. 3 (a) and (b) for two compounds. The Kohn-Sham DFT gives the ground state energy of the static lattice $\frac{3}{3}$ But, in a solid, the nuclei being quantum particles the vibrations exist even at zero temperature $\mathrm{s}^{3}$ Thus, this zero-point energy of the lattice ignored by the KS DFT is obtained using PHONOPY in the harmonic crystal approximation. ${ }^{3}$ The values of zero-point energies obtained in the present study for both the compounds with the help of four functionals are tabulated in Table 2. In the two figures, the intercepts to the $\mathrm{y}$-axis from the Helmholtz free energy curve represent the zero-point energy ${ }^{4.21}$ From the plots it can be observed that the zero-point energies are $\sim 21 \mathrm{~J}$ and $\sim 16$ $\mathrm{J}$ for $\mathrm{Fe}_{2} \mathrm{VAl}$ and $\mathrm{Fe}_{2} \mathrm{TiSn}$, respetively. The reason for this decline in the values of zero-point energy is again related with higher and lower frequencies of the $\mathrm{Fe}_{2} \mathrm{VAl}$ and $\mathrm{Fe}_{2}$ TiSn phonons,respectively. Because, the equational form of zero-point energy is $\frac{1}{2} \sum_{\mathbf{q i}} \hbar \omega_{\mathbf{q} j}, 4,21$. Thus, the proper explanation of the variation $C_{V}$ calculated for both the compounds justify the obtained phonon energy spectrum. Using the thermodynamical relations, $C_{V}$ and $F$ are calculated as functions of temperature based on the equations:

$$
\begin{array}{r}
C_{V}=\sum_{\mathbf{q} \mathbf{j}} k_{B}\left(\frac{\hbar \omega_{\mathbf{q} \mathbf{j}}}{k_{B} T}\right)^{2} \frac{\exp \left(\hbar \omega_{\mathbf{q j}} / k_{B} T\right)}{\left[\exp \left(\hbar \omega_{\mathbf{q} \mathbf{j}} / k_{B} T\right)-1\right]^{2}},(1) \\
F=\frac{1}{2} \sum_{\mathbf{q} \mathbf{j}} \hbar \omega_{\mathbf{q} \mathbf{j}}+k_{B} T \sum_{\mathbf{q} \mathbf{j}} \ln \left[1-\exp \left(-\hbar \omega_{\mathbf{q} \mathbf{j}} / k_{B} T\right)\right](2)
\end{array}
$$

Where, $k_{B}$ is Boltzmann constant, $\hbar$ is reduced Planck's constant, $\omega_{\mathbf{q} \mathbf{j}}$ is phonon frequency for mode $\mathbf{q}, \mathbf{j}$ and $T$ is absolute temperature.

The Debye temperature $\Theta_{D}$ is defined as the temperature above which all modes begin to be excited, and

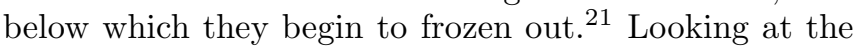
phonon DOS in Fig. 2, the highest energy phonons 
correspond to $\sim 55 \mathrm{meV}$ and $\sim 45 \mathrm{meV}$ for $\mathrm{Fe}_{2} \mathrm{VAl}$ and $\mathrm{Fe}_{2} \mathrm{TiSn}$, respectively based on which the $\Theta_{D}$ for two compounds are $\sim 660 \mathrm{~K}$ and $\sim 540 \mathrm{~K}$. Thus, above these temperatures all the phonons are excited in the crystal lattice. In Fig. 4 (a) and (b), it can be observed that near these temperatures the $C_{V}$ is approaching Dulong and Petit limit. Which indicates that all the phonons starts to contribute to the $C_{V}$ which justifiy the calculated phonon dispersion for two compounds.

\section{Density functionals and phonon properties}

In first-principles DFT calculations the total energy of a crystal is calculated as the sum of electrostatic energy, kinetic energy and exchange-correlation energy ${ }^{3}$ In the direct method, the total energy of the crystal is expanded as a product of force constant matrices and displacements. ${ }^{24}$ Hence, in the harmonic theory of crystals the frequencies, in turn, energy of phonons should depend on the $\mathrm{XC}$ energy used to obtain the total energy. The phonons dispersion plots of $\mathrm{Fe}_{2} \mathrm{VAl}$ obtained from LDA, PBE, PBEsol, and SCAN are shown in Fig. 1 (a)-(d), respectively. It can be noticed that, the phonon branches in PBE obtained vibrational spectrum are the mosted shifted down in energy scale by $\sim 3 \mathrm{meV}$ from that of SCAN and LDA. The energies of phonon branches are nearly similar in case of LDA and SCAN meta-GGA with a difference that 6 optical branches are starting from same point at $35 \mathrm{meV}$ in case of SCAN and while two degenerate branches are startig from $\sim 35$ and $\sim 40 \mathrm{meV}$. While, in general, the PBEsol is producing the spectrum with energy intermediate to those from PBE and LDA (or SCAN). Observing the general features of the phonon bands for $\mathrm{Fe}_{2} \mathrm{VAl}, \mathrm{PBE}$ and SCAN spectra are similar with three degenerate points at $\sim 47 \mathrm{meV}, \sim 33 \mathrm{meV}$ and $0 \mathrm{meV}$ at the $\Gamma$-point. For LDA and PBEsol dispersion curves there is one more additional degenerate point $\sim 40$ $\mathrm{meV}$ and $\sim 33 \mathrm{meV}$, repectively. These observations are well reflected in the phonon DOS of the compound in Fig. 2 (a). DOS curves from PBE are forming the lower bound but showing highest DOS in $\sim 20-40 \mathrm{meV}$ region. These high values of peaks indicate that within the branches seperation is very less because of the low frequency of the phonons produced in PBE calculations. The SCAN or LDA functionals corresponding DOS curves, in general, forming the upper bound. While PBEsol phonon DOS is intermediate that of the three functionals lying

TABLE II: The zero-point energy in joule $(J)$ for two compounds with four different functionals.

\begin{tabular}{lcc}
\hline \hline Functional & $\mathrm{Fe}_{2} \mathrm{VAl}$ & $\mathrm{Fe}_{2} \mathrm{TiSn}$ \\
\hline LDA & 20.99 & 16.87 \\
PBE & 20.09 & 16.02 \\
PBEsol & 20.67 & 16.63 \\
SCAN & 21.04 & 16.95 \\
\hline \hline
\end{tabular}

in between PBE and LDA(or SCAN). Fig. 1 (e)-(h) show the phonon dispersion plots for $\mathrm{Fe}_{2} \mathrm{TiSn}$ compound from four functionals under study. In this case also, similar to the former compound, the PBE produced branches are the most shifted down in energy scale. This shift from the LDA and SCAN produced dispersions is $\sim 1 \mathrm{meV}$, which is to be noted that less than that in the former compound. The phonon branches from PBEsol functional is showing energy intermediate to those of SCAN and LDA. The general features of the four dispersion curves appears to be similar in case of this compound unlike the case of $\mathrm{Fe}_{2} \mathrm{VAl}$. These observations are neatly justified in the phonon DOS plot shown in Fig. 2 (b). As in the case of the former compound, PBE is forming the lower bound in the phonon energy spectrum and showing the the highest value of DOS peaks in the $\sim 10-22 \mathrm{meV}$ and $\sim 25-40 \mathrm{meV}$ regions. The lowest peaks corresponds to the SCAN functional while LDA and PBEsol phonon DOS peaks are lying in between in the DOS peaks. But, in the enrergy, in general, SCAN and LDA are forming the upper bounds. From Fig. 4 (a) and (b), it can be seen that change in XC functionals has negligible effect on $C_{V}$, while in case of $\mathrm{F}$, there is small variation in higher temperatures region for the two compounds.

\section{CONCLUSIONS}

In the present work, along with first-principles DFT to calcuate forces, phonon calculations using finitedisplacement method and supercell approach are used for the study of $\mathrm{Fe}_{2} \mathrm{VAl}$ and $\mathrm{Fe}_{2}$ tiSn compounds. The study is carried out using four XC functionals viz., LDA, PBE, PBEsol and SCAN. Under the harmonic approximation of crystals, phonon dispersion and DOS, PDOS are calculated. Using the thermodynamic relations and obtained phonon frequencies constant-volume specific heat, Helmholtz free energy as functions of temperature, zeropoint energy and Debye temperatures are calculated. The obtained phonon frequencies, justify the classical Dulong and Petit limit constant-volume specific heat at higher temperatures. By comparing the phonon disperion plots and DOS from different functionals, $\mathrm{PBE}$ is found to give the lowest phonon energies while LDA and SCAN are giving higer values of energies. The PBE obtained dispersion energies are $\sim 2 \mathrm{meV}$ and $\sim 1 \mathrm{meV}$ lower than the LDA or SCAN values, respectively. The zeropoint vibrational energy obtained from different functionals are in the order $\mathrm{SCAN}>\mathrm{LDA}>\mathrm{PBEsol}>\mathrm{PBE}$ for both the compounds The value of $\Theta_{D}$ are $\sim 660 \mathrm{~K}$ and $\sim 540 \mathrm{~K}$ for the first and second compound, respectively. Slope of the acoustic branches give sound velocity which is proportional to lattice part of thermal conductivity. By examining the slopes of the acoustic branches of the two compounds, for $\mathrm{Fe}_{2} \mathrm{VAl}$ higher value and $\mathrm{Fe}_{2} \mathrm{TiSn}$ lower value of lattice thermal conductivity is predicted. This qualitative prediction of our work is in agreement with nature of experimental lattice thermal conductiv- 
ity measurements of Leu et. al for both the compounds. Phonon spectra obtained from PBE and SCAN have similar general features while those from LDA and PBEsol have resembling features for $\mathrm{Fe}_{2} \mathrm{VAl}$, while this trend is not observed for the compound $\mathrm{Fe}_{2} \mathrm{TiSn}$.

\section{ACKNOWLEDGEMENTS}

The authors thank Science and Engineering Research Board (SERB), Department of Science and Technology,
Government of India for funding this work. This work is funded under the SERB project sanction order No. EMR/2016/001511.
* Electronic mail: shastri1992@gmail.com

1 W. Kohn and L. J. Sham Phys. Rev., vol. 140, pp. A1133A1138, 1965.

2 J. M. Skelton, D. Tiana, S. C. Parker, A. Togo, I. Tanaka, and A. Walsh The Journal of chemical physics, vol. 143, p. 064710, 2015 .

3 P. Hao, Y. Fang, J. Sun, G. I. Csonka, P. H. Philipsen, and J. P. Perdew Physical Review B, vol. 85, p. 014111, 2012.

4 A. Togo and I. Tanaka, "First principles phonon calculations in materials science," Scripta Materialia, vol. 108, pp. 1-5, 2015.

5 Y. Wang, S.-L. Shang, H. Fang, Z.-K. Liu, and L.-Q. Chen npj Computational Materials, vol. 2, p. 16006, 2016.

6 Y. Nishino, S. Deguchi, and U. Mizutani Phys. Rev. B, vol. 74, p. 115115, 2006.

7 S. Yabuuchi, M. Okamoto, A. Nishide, Y. Kurosaki, and J. Hayakawa Applied Physics Express, vol. 6, p. 025504, 2013.

8 I. Galanakis, P. H. Dederichs, and N. Papanikolaou Phys. Rev. B, vol. 66, p. 174429, 2002.

9 C.-S. Lue and Y.-K. Kuo Journal of applied physics, vol. 96, no. 5, pp. 2681-2683, 2004.

10 C. S. Lue and Y.-K. Kuo Physical Review B, vol. 66, no. 8, p. 085121, 2002.

11 G. Mahan Journal of applied physics, vol. 65, no. 4, pp. 1578-1583, 1989.

\section{REFERENCES}

12 G. Mahan APL Materials, vol. 4, no. 10, p. 104806, 2016.

13 S. S. Shastri and S. K. Pandey Computational Materials Science, vol. 143, 2018.

14 J. P. Perdew and Y. Wang Phys. Rev. B, vol. 45, p. 13244, 1992.

15 J. P. Perdew, K. Burke, and M. Ernzerhof Phys. Rev. Lett., vol. 77, pp. 3865-3868, 1996.

16 J. P. Perdew, A. Ruzsinszky, G. I. Csonka, O. A. Vydrov, G. E. Scuseria, L. A. Constantin, X. Zhou, and K. Burke Phys. Rev. Lett., vol. 100, p. 136406, 2008.

17 J. Sun, A. Ruzsinszky, and J. P. Perdew Phys. Rev. Lett., vol. 115, p. 036402, 2015.

18 P. Blaha, K. Schwarz, G. K. H. Madsen, D. Kvasnicka, and J. Luitz, "wien2k," An augmented plane wave+ local orbitals program for calculating crystal properties, 2001.

19 J. M. Skelton, S. C. Parker, A. Togo, I. Tanaka, and A. Walsh Physical Review B, vol. 89, p. 205203, 2014.

20 D. Do, M.-S. Lee, and S. D. Mahanti Phys. Rev. B, vol. 84, p. 125104, 2011.

21 N. Ashcroft and N. Mermin, Solid State Physics. Cengage Learning, 2011.

22 V. Kanchana, G. Vaitheeswaran, Y. Ma, Y. Xie, A. Svane, and O. Eriksson Phys. Rev. B, vol. 80, p. 125108, 2009.

23 E. Maciá, Thermoelectric Materials: Advances and Applications. CRC Press, 2015.

24 R. Yu, D. Singh, and H. Krakauer Physical Review B, vol. 43, p. 6411, 1991. 